\section{Reducing the stigma of mental illness: a report from a Global Programme of the World Psychiatric Association}

Authors: Norman Sartorius \& Hugh Schulze Publisher: Cambridge University Press, Cambridge, 2005

ISBN: 9780521549431 ; hardcover; 264 pages; price $\mathrm{f} 30$

Stigma has become an ever more frequent answer to many questions about the social burden and psychological suffering associated with major chronic health problems. Mental illnesses figure prominently among them, and this book, focusing on schizophrenia, is concerned with what can and should be done about it. Because populationbased programmes for mental health are sorely needed but less common than those for other areas of public health, experience on the practical implications of community contexts of mental illness is most welcome. Recent public health initiatives on mental health have only begun to complement the emphasis on psychiatric treatment services by focusing attention on preventing mental illness and promoting mental health. Stigma has been identified as a hidden aspect of the burden of mental illness. The efforts described in this book have as their objective the relief of this burden, through mobilizing family and community support and appropriate use of existing mental health services.

This global programme against the stigma of schizophrenia that is the subject of the book was initiated by Norman Sartorius in 1996, when he began his three-year term as president of the World Psychiatric Association (WPA), and is continuing under his guidance as its scientific director. The book chronicles the efforts to establish the programme and describes the conceptual framework that was developed to guide action agendas in diverse national and local settings. These agendas are based on a formulation of stigma as a series of dynamic processes affecting persons with schizophrenia, their families, and health systems that care for them. Having previously served as director of WHO's Division of Mental Health for 17 years, and as an outstanding contributor to the field of mental health, Sartorius was uniquely qualified to identify and respond to the pressing needs to relate cross-cutting global interests and local priorities. The WPA initiative to reduce schizophrenia-related stigma appears to have had substantial impact, motivating public health actions to improve the mental health of populations. This effort is arguably as timely today as the international pilot study on schizophrenia, also led by Sartorius, was four decades ago.

The book provides an informative catalogue of strategies and approaches embodied in various anti-stigma programmes throughout the world. These include organizational activities, research, and interventions aimed at those who stigmatize and those who are the targets of stigma. The bulk of the book details such activities from campaigns carried out in 19 countries, each classified at one of four stages of their development. The interventions described focus on priorities and approaches to influence journalists, students, employers and employees, clergy, police and the criminal justice system, family and community groups, and so forth.

To ensure that the lessons of these various country programmes may effectively guide others interested in establishing similar activities, the authors include a chapter that distils the key campaign elements with respect to target groups and the content of interventions. The power and influence of the mass media are an important focus of programme interests, but are recognized by the authors as being two-edged: programme activities typically focus on making appropriate use of the media to counter stigma; however, left on its own, the media may generate more problems than solutions by supporting stereotypes that perpetrate stigma. An afterword, written by Hugh Schulze, a coauthor with expertise in marketing communications who has been involved in the programmes over the past decade, blames the media for misconceptions, "with so many simple-minded messages that lodge in our minds like stones in our shoes."
In an attempt to classify community concepts of stigma, a concluding section discusses four dimensions of stigma, based on focus group data from studies in Germany that are regarded as widely applicable. These dimensions are (i) stigma arising in the context of interpersonal relations; (ii) structural discrimination that limits access to work, insurance, and other opportunities; (iii) public images of mental illness; and (iv) access to social roles. This section and others dealing with the concept of stigma and analysis of cultural differences (Appendix 2) are less carefully developed, however, than the practical content presented in Appendix 1. Although the structure of this concluding section is confusing (with embedded appendixes to appendixes), it nevertheless constitutes a treasure trove of useful guidelines for developing and implementing anti-stigma programmes.

The importance of acknowledging and reducing the stigma of mental illness is an indisputable premise of the book and the WPA global programme, and the broad action agenda that the book presents to stimulate other programmes with similar aims is a major contribution to efforts to improve the mental health of individuals and populations. In the interplay of stigma and disability, however, an exclusive focus on stigma may neglect other aspects of schizophrenia that are also relevant for community mental health. Stigma is not the only barrier to opportunities for people with this disabling disorder, and an exclusive focus on social engineering to eliminate stigma clearly does not constitute a comprehensive public mental health agenda. Though beyond the scope of this book, the need to distinguish the limitations of disability from the impact of stigma is an essential and inescapable task for people with serious mental illness, their families and professional care-givers, as well as for policy-makers.

Notwithstanding this limitation, the activities of the WPA programme and the motivation and guidelines for other anti-stigma campaigns provided in this book are important. Its publication should strengthen needed activities in this area, and for those who would like to involve themselves in this effort, 
the book will show them how to proceed. It is a valuable resource for mental health professionals and for all who are concerned directly with reducing the hidden burden imposed by the stigma of schizophrenia. The book will also be of use to those involved in other areas of public health, who can draw from the experiences gained in mitigating the stigma of schizophrenia and adapt and apply them to tackling other health problems that are targets of stigma.

\section{Mitchell G Weiss ${ }^{\text {a }}$}

\section{Obesity prevention and public health}

Editors: David Crawford \& Robert W Jeffery

Publisher: Oxford University Press, Oxford, 2005

ISBN: 978-0-19-856600-7; hardcover,

352 pages; price $\mathrm{f} 49.50$

This book is both depressing and exciting. Depressing because it lays bare the inability of public health practitioners to respond adequately to the obesity pandemic. We have been content to observe its emergence and entrenchment in wealthy countries over the last three decades and more recently in low- and middle-income countries. Only in the last 5 years has the public health community become seriously engaged with the problems presented by obesity to the health of populations, and especially poor populations. The excitement comes when the contributing authors become less academic and begin to consider the opportunities for addressing the environmental causes of the obesity pandemic — regrettably this only comes towards the end of the book where the last three (out of fourteen) chapters begin to get serious about the public health options with a real chance of success.

I read the book in a variety of obesegenic environments: an Indian Ocean atoll, a Beijing hotel, and a longhaul air flight. In each of these locations excessive amounts of food (mostly buffets) were readily available (and with no price disincentive since it was included in the "room rate") and the natural environments were not conducive to serious physical activity - the temperature on the atoll and the pollution and traffic in Beijing. At least my physical activity options were not immediately harmful to my health. During my Beijing visit, 20 middle-school children and their teacher were killed by a truck while out jogging in the early morning. Even if physical activity is only a partial answer to obesity, it must be safe.

The focus of the book — said by one of the contributors to be the first of its kind - is inevitably on the USA, both because this is where the obesity epidemic has achieved the greatest levels and received the most media attention and also where the surveillance data are the most complete. However, even for the USA the available data are grossly insufficient to identify the relative contributions of trends in food intake and physical activity pattern to the obesity epidemic. It is clear, however, that the most important contribution of obesity to the global burden of ill-health will occur in low- and middle-income countries. In almost all these countries the epidemic is rapidly taking hold driven by the nutrition transition and even more rapid changes in urban design, especially work and transportation patterns. In some parts of Africa and Asia the situation is complicated by the coexistence of obesity and underweight, sometimes in the same family.

Despite the academic nature of many of the chapters (the book has a total of 1200 references) it is clear that increases in energy intake are the major factor responsible for the rise of obesity. Furthermore, this increase is driven by environmental forces, not the free choices of individuals, especially in childhood. Thus, major changes will be required from the food industries to ensure the global availability of products with a marked reduction in levels of fats, sugar and salt. WHO is in dialogue with the food and non-alcoholic beverages industries, and similar discussions have been held, not only in wealthy countries, but also recently in India and China. The positive statements made by multinational and national companies in this respect will need to be taken up by small-scale companies and systems set in place to monitor the promises of the industry. Major contributions will also be required from other sectors, notably marketing and advertising, education, trade, transport and urban design.

A key issue to be resolved in all countries is the balance of responsibility between individuals and the environments for creating the obesity epidemics. In most of the book a "balanced" approach is predominant. Only in the last three chapters, and appropriately in my view, is the overwhelming role of the environment stressed. It was these chapters that captured my enthusiasm and gave me hope for the future. They deal with the power of litigation to set the terms of engagement; a case study on efforts in Tonga to reduce the importation of fatty foods; and, especially, the agenda for action, despite incomplete evidence for effectiveness, laid out in the final chapter.

So far no country has achieved any notable success in the fight against obesity. Small-scale successes have been achieved in some settings, notably schools and workplaces. But the rise of obesity has yet to show any signs of slowing. Perhaps it will be China that will lead the way — the traditional diet is still entrenched for the majority of the population, "super sizing" has not yet swept the country, and once the authorities get to grips with the problem, positive changes may be possible in a short time.

Robert Beaglehole ${ }^{b}$

\footnotetext{
a Department of Public Health and Epidemiology, Swiss Tropical Institute, Basel, Switzerland (email: mitchell-g.weiss@unibas.ch).

b Chronic Diseases and Health Promotion, World Health Organization, 1211 Geneva 27, Switzerland (email: beagleholer@who.int).
} 